\title{
Color Extraction from Lyrics
}

\author{
Gen Hori \\ Faculty of Business Administration \\ Asia University \\ Tokyo 180-8629, Japan \\ hori@asia-u.ac.jp
}

\begin{abstract}
We establish a procedure for color extraction from lyrics based on the distributed semantic representation obtained by word2vec. To extract colors from lyrics, we exploit the fact that the cosine similarities between words and color names tend to reflect the actual colors of the things the words indicate. We select important words that characterize the lyrics according to the tf-idf statistic and estimate the colors of the important words using the cosine similarities between the words and color names. For evaluation of the color extraction procedure, we implement a software system that automatically generate images based on the colors extracted from given English lyrics. We conduct a subjective evaluation of the color extraction procedure using the software system and the lyrics of three Beatles songs.
\end{abstract}

\section{CCS CONCEPTS}

- Computing methodologies $\rightarrow$ Natural language processing.

\section{KEYWORDS}

natural language processing, word2vec, tf-idf, color, lyrics

\section{INTRODUCTION}

From the viewpoint of artificial intelligence and music creation, although there have been an enormous amount of works on automatic composition and lyrics generation[1][2][3][4], almost no work has been done on automatic cover art generation in spite of its importance and relevance to music production. The purpose of the present study is to introduce a procedure of color extraction from lyrics exploiting the distributed semantic representation obtained by the word2vec algorithm [5][6] with a view of future development of automatic cover art generation systems. We start with an observation that the cosine similarities between words and color names tend to reflect the actual colors of the things the words indicate. Based on the observation, we can estimate the color of a given word by calculating the cosine similarities between the given word and color names and choosing the color that gives the largest value of the cosine similarity. At the same time, we can select important words that characterize the lyrics using the classical tf-idf (term

Permission to make digital or hard copies of part or all of this work for personal or classroom use is granted without fee provided that copies are not made or distributed for profit or commercial advantage and that copies bear this notice and the full citation on the first page. Copyrights for third-party components of this work must be honored.

For all other uses, contact the Owner/Author.

CACRE '19, July 19-21, 2019, Shenzhen, Chin

(C) 2019 Copyright is held by the owner/author(s).

ACM ISBN 978-1-4503-7186-5/19/07.

https://doi.org/10.1145/3351917.3351991 frequency-inverse document frequency) statistic[7]. By combining these two approaches, we can extract the important colors that characterize the lyrics by selecting important words from the lyrics using tf-idf and estimating the colors of the important words exploiting word2vec. Although we use English lyrics to illustrate our method in the present study, the color extraction procedure readily extends for other languages if an adequate corpus and basic color names are given in the language because the procedure does not exploit any specific language structure. Furthermore, for evaluation of the color extraction procedure, we implement a software system that automatically generate images based on the colors extracted from given English lyrics and conduct a subjective evaluation using the software system.

The rest of the paper is organized as follows. Section 2 introduces the color extraction procedure exploiting word2vec and tf-idf. Section 3 describes our software system for evaluation of the color extraction procedure and the result of a subjective evaluation using images generated from the lyrics of three Beatles songs. Section 4 concludes the paper.

\section{COLOR EXTRACTION}

\subsection{Distributed semantic representation}

The distributed semantic representation is a method of embedding words into a vector space. Comparing to the one-hot representation of words, it embeds words into a vector space of a relatively lower dimension of a few hundred. If two words are semantically close to each other, then the corresponding two vectors are close to each other in the vector space. The word2vec algorithm introduced by Mikolov[5][6] is widely used for learning the distributed semantic representation from a corpus. It has been reported that recently developed methods exploiting word2vec outperform previous methods in many natural language processing tasks. We denote the vectors obtained by word2vec representing two words $w_{i}, w_{j}$ by $\boldsymbol{w}_{i}, \boldsymbol{w}_{j}$. Then the cosine similarity, or the cosine of the angle between the two vectors,

$$
\operatorname{cs}\left(\boldsymbol{w}_{i}, \boldsymbol{w}_{j}\right)=\frac{\boldsymbol{w}_{i} \cdot \boldsymbol{w}_{j}}{\sqrt{\left\|\boldsymbol{w}_{i}\right\| \cdot\left\|\boldsymbol{w}_{j}\right\|}}
$$

reflects the semantic similarity of the two words; it takes a large value when the two words are semantically close to each other. The semantic similarity between two words is simply defined in word2vec as the probability of the appearance of two words in proximity in the corpus. Then the cosine similarity is a measure 
of the appearance of two words in proximity. In the present study, we use Mikolov's own implementation of word2vec written in $\mathrm{C}$ and the text 8 corpus ${ }^{1}$ accompanied in the distribution of the implementation for learning. Applying word2vec to the text 8 corpus produces the distributed semantic representations of 75761 English words.

\subsection{Cosine similarity with color names}

We extract colors from lyrics based on an observation that the cosine similarities between words and color names tend to reflect the actual colors of the things the words indicate. This is a natural consequence of the fact that the cosine similarity is a measure of the appearance of two words in proximity in the corpus. Table 1 gives the values of the cosine similarities of three words ("fire," "grass" and "sky") with three color names ("red," "green" and "blue"). For example, the cosine similarity of "sky" with "blue" is larger than that with "red" or "green", which follows the fact that the phrase "blue sky" appears more often than "red sky" or "green sky" in the corpus. Similarly, the cosine similarity of "fire" with "red" and that of "grass" with "green" give the largest values in the first and second rows of Table 1, respectively. We should notice, however, that this does not always hold true. For example, in Table 2, the cosine similarity of "river" with "red" is larger than that with "green" or "blue" following the fact that there are many rivers called "red river" in the world although the actual colors of such rivers are not literally red but closer to blue or green.

Table 1: The cosine similarities between words and color names

\begin{tabular}{c|ccc}
\hline & "red" & "green" & "blue" \\
\hline "fire" & 0.146 & 0.105 & 0.098 \\
"grass" & 0.201 & 0.358 & 0.259 \\
"sky" & 0.200 & 0.235 & 0.418 \\
\hline
\end{tabular}

Table 2: A counterexample in the cosine similarity and the actual color

\begin{tabular}{c|ccc}
\hline & "red" & "green" & "blue" \\
\hline "river" & 0.194 & 0.115 & 0.090 \\
\hline
\end{tabular}

Based on the assumption that such counterexamples are few, we estimate the color of a given word by calculating the cosine similarities of the word with color names and choosing the color that gives the largest value of the cosine similarity.

\subsection{Selection of important words}

To extract colors that describe the lyrics, we select important words that characterize the lyrics and then estimate the colors of the

\footnotetext{
${ }^{1}$ The first $10^{8}$ bytes of the cleaned text data extracted from the English Wikipedia dump on Mar. 3, 2006
}

selected words. The tf-idf (term frequency-inverse document frequency) [7] is a statistic widely used for estimating how important a word $w_{i}$ is to a document $d_{j}$ in a set of documents $D$. The tf-idf of the word $w_{i}$ to the document $d_{j}$ is defined as a product of the term frequency and the inverse document frequency,

$$
t f_{i, j} \times i d f_{i}
$$

where the term frequency is defined as,

$$
t f_{i, j}=\frac{n_{i, j}}{\sum_{k} n_{k, j}}
$$

where $n_{i, j}$ is the frequency of the word $w_{i}$ in the document $d_{j}$, and the inverse document frequency is defined as,

$$
i d f_{i}=\log \frac{|D|}{\left|\left\{d \mid w_{i} \in d\right\}\right|},
$$

where $|D|$ is the total number of the documents in the set $D$ and $\left|\left\{d \mid w_{i} \in d\right\}\right|$ is the number of the documents in $D$ that contain the word $w_{i}$. The inverse document frequency is used to reduce the score of common words. We sort the words in lyrics according to the values of tf-idf and select the top words for color extraction from lyrics.

\subsection{Color extraction from lyrics}

For color extraction from lyrics, we use nine fundamental color names, "black," "gray," "white," "red," "green," "blue," "cyan," "magenta" and "yellow," which are the vertices and the center of the RGB color cube. We calculate the cosine similarities of each word in lyrics ${ }^{2}$ with the nine fundamental colors and estimate that the color of the word is the one that gives the largest value of the cosine similarity. If the largest value is less than a predetermined thresh$\mathrm{old}^{3}$, we estimate that the word is colorless. We sort all the colored words in descending order of $\mathrm{tf}$-idf and name the colors of the top words as the extracted colors from the lyrics. The color extraction procedure readily extends for other languages if an adequate corpus and basic color names are given in the language because tf-idf and word2vec do not use any specific language structure.

\section{SOFTWARE SYSTEM FOR EVALUATION}

\subsection{Implementation}

We implement a sofware system that automatically generate images from English lyrics based on the color extraction procedure. It extracts the primary and secondary colors from given English lyrics and generate an image based on the two extracted colors. The primary color is the color of the top word in the list of the colored words in the descending order of tf-idf. The secondary color is the color of the second word. To generate images, we use the ImageMagick library, a standard library for image generation and format conversion. Specifically, we give the primary and secondary colors as the inputs to the library and choose plasma option of the library to generate images like oil paintings.

\footnotetext{
${ }^{2}$ We remove stopwords before we calculate the cosine similarity in our software system.

${ }^{3}$ We set the threshold to 0.1 in our software system.
} 


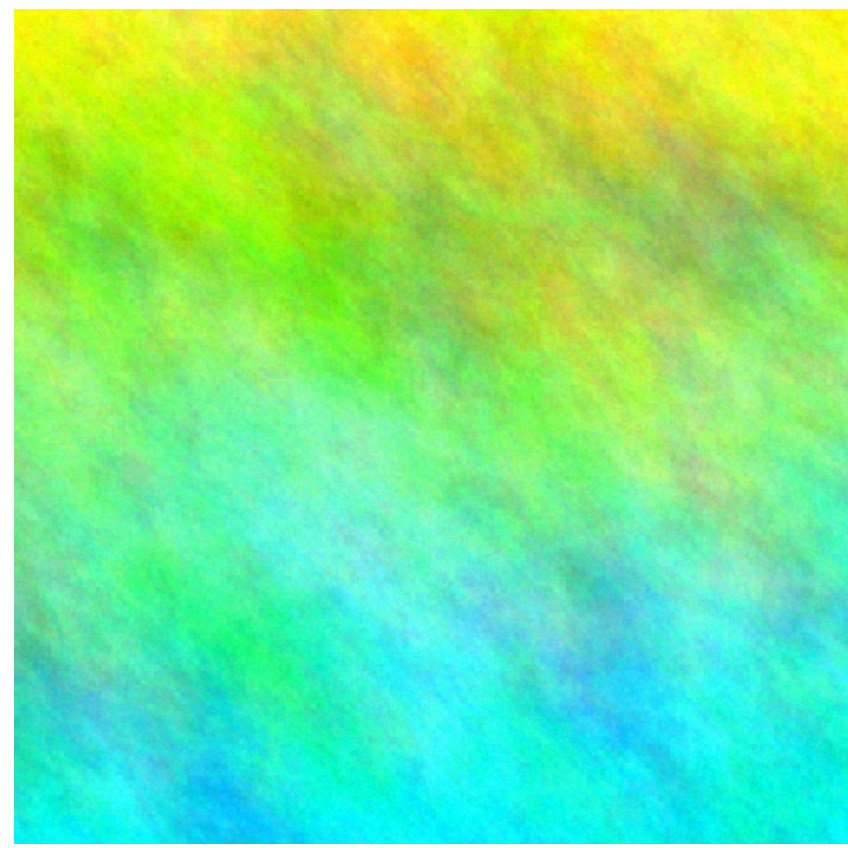

Figure 1: An image generated from the lyrics of "Let It Be"

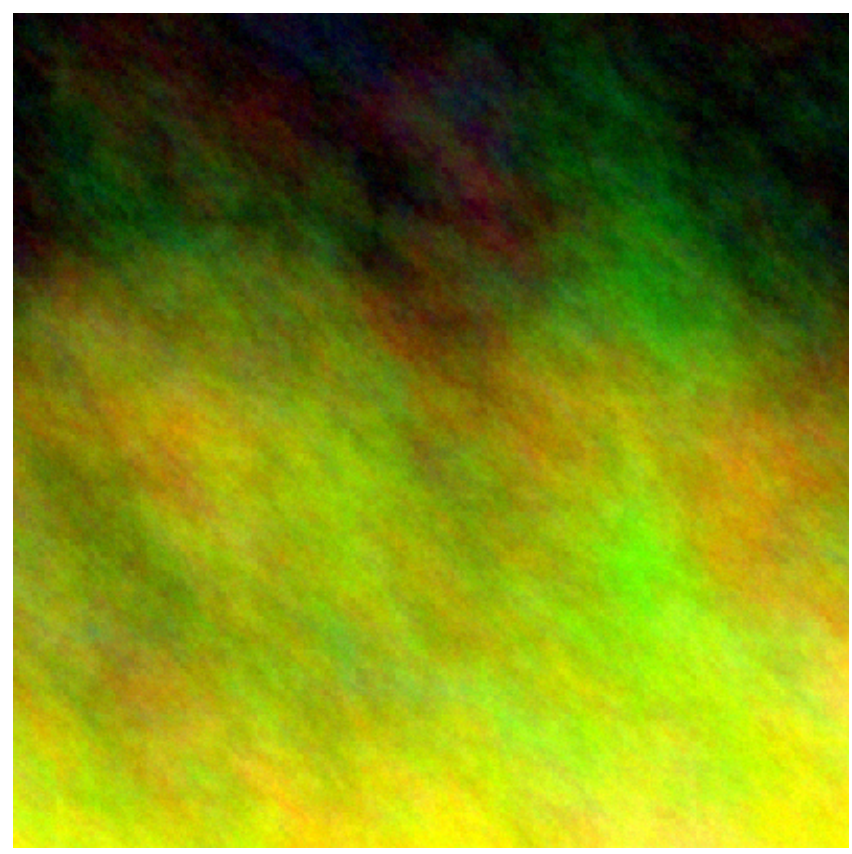

Figure 2: An image generated from the lyrics of "Helter Skelter”

\subsection{Examples}

Figures 1-3 exhibit images automatically generated by our software system from lyrics of three Beatles songs, "Let It Be," "Helter Skelter" and "Free As A Bird." Tables 3-5 list the tf-idf, the estimated colors and the cosine similarities with color names used in generating the

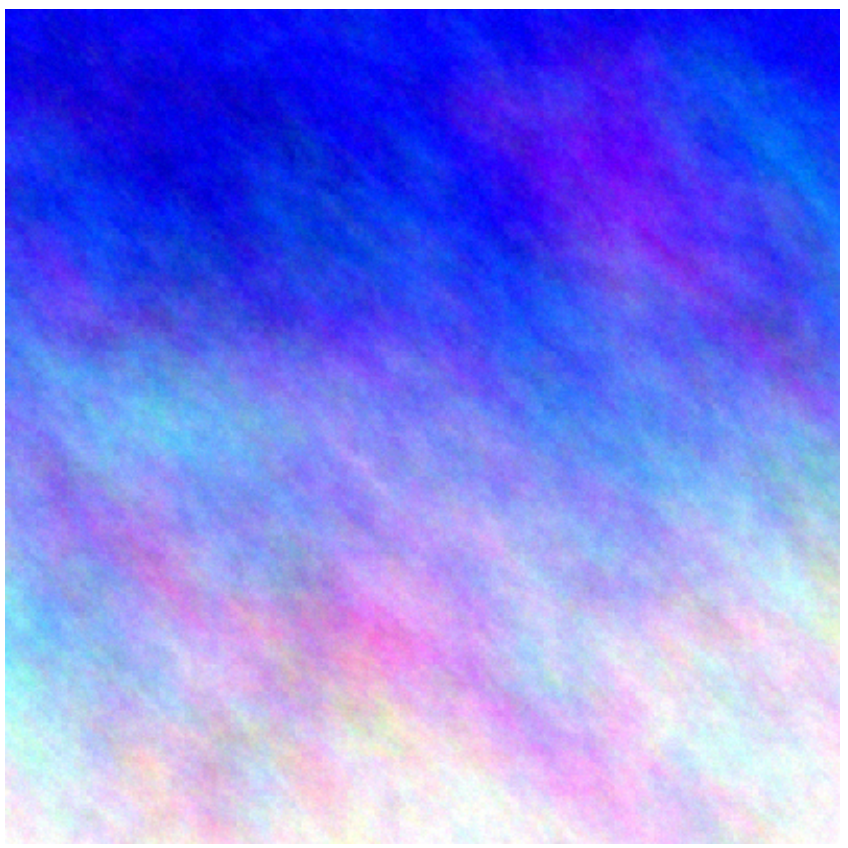

Figure 3: An image generated from the lyrics of "Free As A Bird”

three images where the values of the cosine similarities displayed in red text are the largest ones that give the estimated colors. In the lyrics of "Let It Be," the word "whisper" has the largest tf-idf among the colored words and the largest cosine similarity with "yellow" which makes the primary color of the lyrics yellow. The word "music" has the second largest tf-idf with a slight margin with the word "wake" and makes the secondary color cyan. In the lyrics of "Helter Skelter," the title word is devided into two which give the same color. If the colors in the first place and the second place are the same, we take the color in the third place as the secondary color. In the lyrics of "Free As A Bird," the primary color is blue by the word "bird" and the secondary color is white by the word "home." We note that the primary color is shared by the words "bird," "wings" and "fly" which are related to each other.

\subsection{Subjective evaluation}

We conducted a subjective evaluation of the generated images and thus the proposed color extraction procedure. A total of 43 subjects in the age range of 18-22 participated in the evaluation, among which 23 are males and 20 females. The subjects looked at the printed lyrics of the three Beatles songs and the three images of Figures 1-3 generated from the lyrics of the three songs, and then answered the following questionnaire. The images were shown to subjects in random order and the subjects answered the questionnaire without knowing the correspondence between the lyrics and the images. They did not listen to the audio of the songs before answering the questionnaire. 
Table 3: The tf-idf and estimated colors of words from the lyrics of "Let It Be"

\begin{tabular}{|c|c|c|c|c|c|c|c|c|c|c|c|}
\hline \multicolumn{3}{|c|}{ color name } & black & gray & white & red & green & blue & cyan & magenta & yellow \\
\hline \multicolumn{3}{|c|}{ color } & & & & & & & & & \\
\hline tf-idf & word & color & \multicolumn{9}{|c|}{ cosine similarity } \\
\hline 0.244 & whisper & & 0.199 & 0.141 & 0.177 & 0.185 & 0.220 & 0.203 & 0.124 & 0.111 & 0.248 \\
\hline 0.075 & music & & 0.070 & -0.108 & 0.069 & -0.120 & -0.055 & 0.044 & 0.101 & -0.088 & -0.017 \\
\hline 0.075 & wake & & 0.066 & 0.068 & 0.047 & 0.173 & 0.093 & 0.117 & -0.017 & 0.003 & 0.074 \\
\hline 0.071 & night & & 0.182 & 0.049 & 0.177 & 0.162 & 0.188 & 0.242 & 0.002 & -0.004 & 0.115 \\
\hline 0.071 & light & & 0.164 & 0.134 & 0.079 & 0.165 & 0.205 & 0.239 & 0.369 & 0.223 & 0.371 \\
\hline 0.069 & see & & 0.059 & -0.021 & -0.008 & 0.007 & 0.104 & 0.116 & 0.155 & 0.081 & 0.060 \\
\hline 0.059 & right & & -0.024 & 0.038 & 0.041 & 0.095 & 0.137 & 0.033 & 0.043 & 0.054 & 0.096 \\
\hline 0.051 & shines & & 0.269 & 0.241 & 0.292 & 0.334 & 0.246 & 0.313 & 0.278 & 0.210 & 0.407 \\
\hline 0.050 & cloudy & & 0.146 & 0.129 & 0.176 & 0.222 & 0.205 & 0.281 & 0.196 & 0.201 & 0.259 \\
\hline 0.047 & parted & & 0.004 & -0.049 & 0.003 & 0.142 & -0.009 & 0.038 & -0.070 & -0.129 & 0.023 \\
\hline 0.046 & shine & & 0.184 & 0.210 & 0.210 & 0.214 & 0.263 & 0.327 & 0.254 & 0.185 & 0.307 \\
\hline 0.042 & tomorrow & & 0.056 & 0.059 & 0.034 & 0.020 & 0.095 & 0.213 & 0.088 & -0.011 & 0.040 \\
\hline 0.036 & darkness & & 0.269 & 0.160 & 0.156 & 0.139 & 0.203 & 0.191 & 0.132 & 0.044 & 0.185 \\
\hline 0.034 & comes & & 0.046 & -0.009 & 0.022 & -0.003 & 0.017 & 0.124 & 0.146 & 0.080 & 0.022 \\
\hline 0.022 & standing & & -0.023 & -0.014 & 0.119 & 0.072 & 0.073 & 0.094 & -0.133 & -0.009 & -0.006 \\
\hline 0.020 & sound & & 0.095 & 0.013 & 0.053 & -0.005 & -0.030 & 0.136 & 0.183 & 0.016 & 0.076 \\
\hline 0.018 & front & & 0.109 & 0.008 & 0.200 & 0.246 & 0.075 & 0.120 & -0.030 & 0.063 & 0.120 \\
\hline
\end{tabular}

Table 4: The tf-idf and estimated colors of words from the lyrics of "Helter Skelter"

\begin{tabular}{|c|c|c|c|c|c|c|c|c|c|c|c|}
\hline \multicolumn{3}{|c|}{ color name } & black & gray & white & red & green & blue & cyan & magenta & yellow \\
\hline \multicolumn{3}{|c|}{ color } & & & & & & & & & \\
\hline tf-idf & word & color & \multicolumn{9}{|c|}{ cosine similarity } \\
\hline 0.526 & skelter & & 0.245 & 0.167 & 0.184 & 0.045 & 0.004 & 0.051 & 0.014 & 0.024 & 0.030 \\
\hline 0.526 & helter & & 0.253 & 0.186 & 0.214 & 0.110 & 0.047 & 0.083 & 0.023 & 0.064 & 0.070 \\
\hline 0.141 & turn & & 0.037 & -0.075 & -0.005 & 0.041 & 0.043 & 0.027 & -0.041 & -0.103 & 0.104 \\
\hline 0.129 & see & & 0.059 & -0.021 & -0.008 & 0.007 & 0.104 & 0.116 & 0.155 & 0.081 & 0.060 \\
\hline 0.129 & top & & 0.119 & 0.012 & 0.094 & 0.191 & 0.122 & 0.190 & -0.023 & -0.030 & 0.122 \\
\hline 0.124 & dancer & & 0.052 & -0.000 & 0.139 & 0.013 & -0.023 & 0.059 & 0.065 & 0.084 & 0.040 \\
\hline 0.096 & bottom & & 0.152 & 0.125 & 0.200 & 0.217 & 0.184 & 0.236 & -0.007 & 0.046 & 0.202 \\
\hline 0.078 & look & & 0.105 & 0.092 & 0.111 & 0.081 & 0.092 & 0.112 & 0.079 & 0.033 & 0.201 \\
\hline 0.074 & slide & & 0.045 & -0.100 & -0.052 & -0.039 & 0.110 & 0.050 & 0.011 & -0.011 & 0.071 \\
\hline 0.067 & hey & & 0.101 & 0.017 & 0.162 & 0.061 & 0.148 & 0.158 & -0.018 & -0.017 & 0.171 \\
\hline 0.065 & ride & & 0.177 & 0.106 & 0.141 & 0.134 & 0.121 & 0.170 & 0.005 & 0.027 & 0.137 \\
\hline 0.061 & head & & 0.099 & 0.113 & 0.126 & 0.139 & 0.046 & 0.136 & -0.093 & 0.049 & 0.041 \\
\hline 0.061 & blisters & & 0.060 & 0.112 & 0.048 & 0.048 & 0.034 & 0.037 & -0.011 & 0.148 & 0.149 \\
\hline 0.034 & till & & 0.119 & 0.122 & 0.012 & 0.105 & -0.009 & 0.043 & 0.055 & 0.095 & 0.056 \\
\hline 0.016 & comes & & 0.046 & -0.009 & 0.022 & -0.003 & 0.017 & 0.124 & 0.146 & 0.080 & 0.022 \\
\hline 0.015 & won & & 0.021 & -0.053 & 0.070 & 0.109 & 0.075 & 0.045 & -0.033 & 0.047 & -0.001 \\
\hline
\end{tabular}


Table 5: The tf-idf and estimated colors of words from the lyrics of "Free As A Bird"

\begin{tabular}{|c|c|c|c|c|c|c|c|c|c|c|c|c|}
\hline \multicolumn{3}{|c|}{ color name } & black & gray & white & red & green & blue & cyan & magenta & yellow \\
\hline \multicolumn{3}{|c|}{ color } \\
\hline tf-idf & word & color & \multicolumn{7}{c|}{ cosine similarity } \\
\hline 1.301 & bird & & 0.136 & 0.193 & 0.153 & 0.192 & 0.118 & 0.240 & 0.035 & 0.105 & 0.097 \\
\hline 0.384 & home & & 0.035 & 0.024 & 0.163 & 0.123 & 0.105 & 0.038 & -0.092 & -0.027 & -0.021 \\
\hline 0.353 & like & 0.135 & 0.063 & 0.109 & 0.043 & 0.093 & 0.108 & 0.039 & -0.068 & 0.214 \\
\hline 0.260 & best & & 0.077 & -0.087 & 0.022 & 0.017 & 0.063 & 0.075 & 0.114 & -0.008 & -0.072 \\
\hline 0.236 & dry & & 0.064 & 0.083 & 0.060 & 0.101 & 0.099 & 0.080 & -0.027 & 0.007 & 0.176 \\
\hline 0.103 & really & & 0.023 & 0.002 & -0.059 & -0.040 & -0.053 & -0.008 & 0.106 & -0.045 & -0.018 \\
\hline 0.093 & wings & 0.119 & 0.220 & 0.146 & 0.250 & 0.240 & 0.333 & 0.067 & 0.107 & 0.214 \\
\hline 0.084 & fly & & 0.051 & 0.112 & 0.098 & 0.122 & 0.121 & 0.213 & -0.023 & 0.045 & 0.107 \\
\hline 0.045 & touch & & 0.165 & 0.183 & 0.176 & 0.154 & 0.187 & 0.168 & 0.204 & 0.106 & 0.254 \\
\hline 0.028 & mean & & 0.029 & 0.058 & 0.024 & -0.022 & 0.051 & 0.078 & 0.180 & 0.094 & 0.138 \\
\hline
\end{tabular}

Table 6: Results of subjective evaluation

\begin{tabular}{c|c|c|c}
\hline & $\begin{array}{c}\text { Fig.1 (Generated from } \\
\text { "Let It Be") }\end{array}$ & $\begin{array}{c}\text { Fig.2 (Generated from } \\
\text { "Helter Skelter") }\end{array}$ & $\begin{array}{c}\text { Fig.3 (Generated from } \\
\text { "Free As A Bird") }\end{array}$ \\
\hline $\begin{array}{c}\text { Q1. (Suitable for } \\
\text { "Let It Be") }\end{array}$ & $61.9 \%$ & $9.5 \%$ & $28.6 \%$ \\
\hline $\begin{array}{c}\text { Q2. (Suitable for } \\
\text { "Helter Skelter") }\end{array}$ & $22.0 \%$ & $58.5 \%$ & $19.5 \%$ \\
\hline $\begin{array}{c}\text { Q3. (Suitable for } \\
\text { "Free As A Bird") }\end{array}$ & $38.1 \%$ & $7.1 \%$ & $54.8 \%$ \\
\hline
\end{tabular}

Q1. Which of the following is most suitable for "Let It Be"? (Single option)

Q2. Which of the following is most suitable for "Helter Skelter"? (Single option)

Q3. Which of the following is most suitable for "FreeAs A Bird"? (Single option)

Table 6 gives the results of the subjective evaluation. First of all, we note that the diagonal elements give the largest values for all the rows, which means that, for each lyrics, the largest number of subjects chose the image generated from the lyrics in question. Also the diagonal elements give the largest values for all the columns, which means that each image was chosen mostly for the lyrics used to generate it. Those results imply that the proposed color extraction procedure works appropriately for the lyrics of those three songs.

\section{CONCLUSION}

We introduced a novel procedure of color extraction from lyrics exploiting tf-idf and word2vec. The procedure is based on the fact that the cosine similarities between words and color names tend to reflect the actual colors of the things the words indicate. We also implemented a software system that automatically generates images from given English lyrics and conducted a subjective evaluation of the color extraction procedure using the software system and the lyrics of three Beatles songs. The results of the evaluation imply that the proposed color extraction procedure works appropriately for the lyrics of those three songs. Our future study plan is to develop a system that automatically generates cover arts from lyrics for which we have two directions. One direction is to employ recently developed text-to-image techniques based on GAN (generative adversarial network) such as StackGAN[8] to generate more concrete images from lyrics. In this direction, the color extraction procedure introduced in the present work will be also useful for setting color tone for generated images. Another direction is to extract colors or other elements from the melodies and chord progressions of given songs and use them for cover art generation.

\section{REFERENCES}

[1] Tommaso Bolognesi. 1983. Automatic composition: Experiments with self-similar music. Computer Music fournal 7, 1 (1983), 25-36. 
[2] Alfonso Ortega de la Puente, Rafael Sánchez Alfonso, and Manuel Alfonseca Moreno. 2002. Automatic composition of music by means of grammatical evolution. In ACM SIGAPL APL Quote Quad, Vol. 32. ACM, 148-155.

[3] Satoru Fukayama, Kei Nakatsuma, Shinji Sako, Takuya Nishimoto, and Shigeki Sagayama. 2010. Automatic song composition from the lyrics exploiting prosody of the Japanese language. In Proc. 7th Sound and Music Computing Conference (SMC). 299-302.

[4] Satoru Fukayama, Kei Nakatsuma, Shinji Sako, Yuichiro Yonebayashi, Tae Hun Kim, Si Wei Qin, Takuho Nakano, Takuya Nishimoto, and Shigeki Sagayama. 2009. Orpheus: Automatic composition system considering prosody of Japanese lyrics. In International Conference on Entertainment Computing. Springer, 309-310.
[5] Tomas Mikolov, Kai Chen, Greg Corrado, and Jeffrey Dean. 2013a. Efficient estimation of word representations in vector space. arXiv preprint arXiv:1301.3781 (2013).

[6] Tomas Mikolov, Ilya Sutskever, Kai Chen, Greg S Corrado, and Jeff Dean. 2013b. Distributed representations of words and phrases and their compositionality. In Advances in neural information processing systems. 3111-3119.

[7] Karen Sparck Jones. 1972. A statistical interpretation of term specificity and its application in retrieval. Journal of documentation 28, 1 (1972), 11-21.

[8] Han Zhang, Tao Xu, Hongsheng Li, Shaoting Zhang, Xiaogang Wang, Xiaolei Huang, and Dimitris N Metaxas. 2017. Stackgan: Text to photo-realistic image synthesis with stacked generative adversarial networks. In Proceedings of the IEEE International Conference on Computer Vision. 5907-5915. 